\title{
Moderating Effect of Satisfaction with Community Child Center Services in the Relationship Between the Abused Experiences and School Adjustment of Children
}

\author{
Yebin Jeon, Soonkyu Kim* \\ Jeonbuk National University, Department of Social Welfare
}

\begin{abstract}
This study examined whether community child centers offer any buffer effect in the relationship between children's abuse experiences and school adjustment, and more specifically, it reviewed the trend of such a change by examining the satisfaction with the services provided by community child centers. To this end, this study used the first survey(2018) data from the community child center third panel, which were collected by the National Youth Policy Institute upon the consignment of the Ministry of Health and Welfare and the Headquarters for Community Child Center. In total, $5364^{\text {th }}$ graders were selected as the subjects of this study, and the data were analyzed using structural equation models such as MARSH in order to identify whether satisfaction had any moderating effect in the relationship between the abuse experiences and school adjustment of children. The results showed that, as in previous studies, abuse experiences had a negative effect on children's school adjustment, while satisfaction with the services of community child centers had a positive effect on school adjustment. In addition, satisfaction with community child centers' services had a moderating effect of reducing the negative effect of abuse experiences on school adjustment, and it was also revealed that although children had abuse experiences, the group of children with a higher satisfaction level with community child centers' services displayed a higher level of school adjustment compared to the group with lower service satisfaction. Based on these results, this study presented a service intervention strategy for community child centers.
\end{abstract}

Keywords: care institution; child welfare; community; school environment; social support 